\title{
Competencias de directores académicos en instituciones de educación superior: Un estudio en el sureste de México
}

\author{
Competencies of Academic Directors at Institutions of Higher Learning: \\ A Study of Southeast Mexico
}

\author{
Roger Manuel Patrón-Cortés ${ }^{1}$ \\ Universidad Autónoma de Campeche \\ Facultad de Contaduría y Administración \\ Campeche, México \\ roger_patron_cortes@hotmail.com
}

Recibido 21 diciembre 2015 • Aceptado 14 enero 2016 • Corregido 04 febrero 2016

\begin{abstract}
Resumen. Este trabajo presenta aspectos críticos relacionados con la formación de directores académicos de una institución de educación superior ubicada en el sureste de México. Asimismo, tiene como objetivo identificar las competencias que utilizan y las que necesitan adquirir con miras a mejorar la eficacia y productividad escolar. El estudio es de corte cualitativo y emplea como método un estudio de campo basado en cuatro casos seleccionados. Se incluyen observaciones, entrevistas y análisis de documentación. Los resultados indican que existen diferencias entre conocimientos y competencias, pues aunque los directores tienen la preparación que les permite estudiar y comprender aspectos relacionados con la educación superior, se les dificulta al momento de aplicarlos. Los directores manejan más las competencias requeridas para el logro de resultados y la unidad interna de la institución, y usan menos las que se refieren a la relación eficaz de la persona consigo misma y con el entorno. En términos generales, los directores requieren adquirir y desarrollar competencias principalmente en las áreas del comportamiento humano, tales como red de relaciones efectivas, negociación, comunicación, empatía, y trabajo en equipo, entre otras. En este sentido, los directores necesitan desarrollar relaciones humanas efectivas tanto con la comunidad escolar como con personas clave del entorno externo para mejora de la eficacia y productividad de la institución.
\end{abstract}

Palabras clave. Administración de la educación superior; gestión de la educación; gestión universitaria; dirección; capital humano

Abstract. This paper looks at critical aspects regarding the professional development of academic directors at an institute for higher learning in Southeast Mexico by identifying the skills and competencies required for improving productivity. The study uses a qualitative approach based on four case studies where results are obtained through observation, interviews and a bibliographical review.

1 Doctor en Ciencias Administrativas, Universidad Anáhuac Mayab, México. Profesor investigador titular de la Facultad de Contaduría y Administración, Universidad Autónoma de Campeche, México. Entre sus reconocimientos se encuentran la Certificación Académica en Administración (ANFECA, 2012-2015), Perfil Deseable (PROMEP, (2012-2015) y es miembro del Sistema Nacional de Investigadores nivel I del CONACYT (2013-2015). Su interés investigativa se centra en el comportamiento organizacional y calidad educativa. Ha presentado resultados de sus estudios en congresos nacionales e internacionales. También ha publicado diversos artículos y capítulos de libro arbitrados. Asimismo, es autor del libro Clima organizacional y satisfacción laboral: una propuesta metodológica. 
Based on the results obtained, the difference between knowledge and competencies lies in its application. Directors may have the necessary academic training regarding administrative concepts, yet may have difficulty in implementing them. They possess the necessary skills for achieving results but overlook those that foster positive relationships with themselves and their environment.

Administrators must develop skills aimed at better understanding human behaviour, building effective relationships, negotiation, communication, empathy and strengthening teamwork with the school community and key actors to improve the efficacy and productivity of the institution.

Keywords. Higher education administration; educational management; university management; leadership; human capital

\section{Introducción}

La dirección escolar y su influencia en la eficacia y productividad de las escuelas, son aspectos que se consideran cada vez más importantes en las políticas educativas de cualquier país. En México, el Programa para la Modernización Educativa y la implementación del Proyecto Escolar hicieron crecer el interés por realizar estudios de la dirección escolar (Poder Ejecutivo Federal, 1996). Asimismo, la mayoría de los estudios de eficacia escolar a través del tiempo, han señalado que detrás de un centro eficaz, subyace la figura del director que ejerce un liderazgo efectivo. El liderazgo no solo descansa sobre la calidad de los rasgos personales sino fundamentalmente en la capacidad para relacionarse con la visión, valores y metas de la escuela y especialmente, en la forma de gestionar el cambio para incrementar la calidad de los aprendizajes en el centro educativo (Sammons, Hillman y Mortimer, 1995). El director como líder requiere poseer ciertas competencias para innovar la actividad educativa, tomar decisiones y comunicarse con autoridades, profesores, padres de familia y estudiantes. Es decir, los directores tienen la difícil tarea de conjugar actividades administrativas y educativas.

Según Uribe (2007), las prácticas de gestión revelan el nivel de competencias de los directivos, pues es competente aquel que cambia los conceptos en acciones a través de las capacidades, habilidades o prácticas que un directivo evidencia en su trabajo diario. Así como los profesores son clave en los procesos de aprendizaje, los directivos son igualmente clave para crear las condiciones institucionales que promueva la eficacia de la organización escolar. Villa (2006) señala que, hasta hace poco, el conocimiento y dominio de la información representaba dos tercios de la valoración profesional de un puesto directivo, pero que actualmente solo representa el $20 \%$ o el $25 \%$, diversificándose esta valoración en otros aspectos tales como destreza en la búsqueda de la información, trabajo en equipo, orientación a resultados, iniciativa y autoaprendizaje, entre otros.

\section{Antecedentes}

El establecimiento de políticas de cobertura internacional para llevar a cabo una profunda transformación de la educación superior en el mundo, ha generado cambios en la educación en América Latina y por lo tanto, en México. Sandoval y Unda (2004) plantean que las condiciones 
de trabajo se han visto impactadas por la diferenciación y diversificación de funciones y actividades académicas, el tamaño de las escuelas y la composición del cuerpo estudiantil, entre otros aspectos. Esta nueva dinámica social, ha generado un ambiente laboral completamente diferente al que se tenía hace treinta años.

El director académico actual se ve en la necesidad de contar con las competencias necesarias para lograr el incremento de la calidad educativa, la eficacia y productividad escolar que los tiempos modernos exigen, pues como señala Elizondo (2011), el director académico juega un papel vital en la calidad de la educación, ya que es el vínculo entre la visión y misión institucionales, y el quehacer de cada día de los profesores y alumnos en el aula, en el laboratorio o en el campo de trabajo.

Este estudio se realizó en una universidad pública ubicada en el sureste de México, en el año de 2015. En las últimas evaluaciones realizadas a esta institución, se detectó que algunas Facultades presentan problemas vinculados con la calidad y la eficacia institucional. De acuerdo con los administradores de la organización, los directores académicos podrían tener cierta influencia en la problemática planteada, por lo que cuatro directores académicos de las Facultades que integran las Dependencias de Educación Superior (DES) de Ciencias Sociales aceptaron participar en el estudio que tiene como objetivo identificar las competencias que utilizan estos directores, así como las que necesitan para mejorar su gestión laboral y contribuir al desarrollo de la institución y de México. Aunque los resultados son válidos solamente para las Facultades de la universidad participante, la metodología puede replicarse en otras instituciones de educación superior con las debidas adecuaciones.

Los resultados contribuirán a enriquecer las investigaciones sobre las competencias directivas que estos profesionales emplean y las que requieren adquirir, pues coadyuvan a comprobar la tesis expuesta por McClelland (1975), citado por Gross (2008), quien indica que los conocimientos adquiridos difieren de las competencias desarrolladas. Además, este estudio permite que, al mejorar y fortalecerse las competencias directivas, se logren beneficios tales como: (a) incrementar la calidad educativa, (b) mayor eficacia y productividad escolar; (c) mejorar las relaciones interpersonales de los involucrados, (d) comunidad educativa más contenta con los servicios ofrecidos, entre otros. Este trabajo presenta la fundamentación teórica relativa al tema, después explica el método de investigación para la recolección de datos, el desarrollo y discusión de los resultados, para finalmente exponer conclusiones y sugerencias de mejoramiento.

\section{Fundamentación teórica}

De acuerdo con Gross (2008), McClelland fue el primero en acuñar el término "competencias", en el año de 1975, al comprobar que los conocimientos y los test de inteligencia no eran capaces de predecir la adaptación de las personas a los problemas de la vida y al éxito profesional. McClelland argumentaba que desempeñar adecuadamente el trabajo dependía 
más de las características propias de la persona que de su currículum, experiencia y habilidades, por lo que investigó sobre nuevas variables que le permitieran establecer una mejor predicción del rendimiento laboral, a las que llamó competencias.

\section{Concepto de competencias}

Spencer y Spencer (1993) señalan que las competencias son características subyacentes en una persona relacionadas a un estándar de efectividad y/o a una actuación superior en un trabajo o situación. Para McClelland (1975), citado por Robbins y Judge (2013), las competencias se refieren a las características subyacentes en un individuo que están vinculadas a los comportamientos y a la acción exitosa en su trabajo, entendiendo por "características subyacentes" a todos los aspectos que la persona tiene y pone al servicio de su comportamiento laboral. Las características subyacentes de cada individuo son el resultado de la suma de sus capacidades cognoscitivas (aptitudes), tendencias de conducta (actitudes), rasgos de personalidad y conocimientos adquiridos, ya sean teóricos o basados en la experiencia.

Las competencias crean un vínculo entre las actividades por ejecutary los comportamientos para hacerlo, por una parte, y las cualidades personales esenciales para comportarse de forma satisfactoria, por otra. Desde este punto de vista, un individuo es competente si puede realizar un trabajo de acuerdo con el estándar y el desempeño esperados.

Cardona y Chinchilla (1999) diferencian entre las competencias técnicas o referidas a un puesto concreto, y las directivas, las cuales son genéricas y se dividen en: (a) estratégicas, (b) intratégicas, y (c) de eficacia personal.

Las competencias estratégicas determinan la capacidad del directivo y sus relaciones con el entorno externo de la organización. Comprenden las siguientes variables:

a) Visión de negocio. El individuo reconoce y hace buen uso de las oportunidades, los peligros y las fuerzas externas que influyen en la competitividad del negocio.

b) Resolución de problemas. Las personas estudian un problema complejo y tienen la capacidad de síntesis y de toma de decisiones.

c) Gestión de recursos. Se usan los recursos de modo eficaz para lograr los objetivos.

d) Orientación al cliente. Responde en tiempo y eficacia a las necesidades del cliente.

e) Red de relaciones efectivas. Desarrolla y sostiene una amplia red de relaciones con personas clave de la empresa y de su entorno.

f) Negociación. Consigue el apoyo y la conformidad de personas y grupos clave que influyen en su área de responsabilidad. 
Las competencias intratégicas se refieren a la capacidad ejecutiva y de liderazgo de un directivo al interior de la institución. Comprenden las siguientes variables:

a) Comunicación. Informa de manera efectiva y aporta evidencias para respaldar sus opiniones y resoluciones.

b) Organización. Asigna funciones a las personas adecuadas para realizar el trabajo y planifica su seguimiento.

c) Empatía. Entiende, tiene en cuenta a los demás y respeta sus sentimientos.

d) Delegación. Trata que su equipo de trabajo tome decisiones para lograr sus objetivos.

e) Coaching. Apoya al personal para que mejorey desarrolle sus habilidades y capacidades.

f) Trabajo en equipo. Establece un clima de colaboración, comunicación y confianza entre los integrantes de su equipo de trabajo y los motiva para el logro de objetivos comunes.

Las competencias de eficacia personal distinguen los hábitos básicos de una persona y la relación entre ella misma y su entorno. Comprenden las siguientes variables:

a) Proactividad. Demuestra iniciativa, creatividad y autonomía personal.

b) Autogobierno. Posee disciplina, concentración y autocontrol.

c) Gestión personal. Mantiene adecuado control del tiempo, estrés y riesgo ante situaciones de gran responsabilidad.

d) Desarrollo personal. Se autoevalúa para lograr cambios de comportamiento que fortalezcan su gestión directiva.

\section{Contexto de la universidad pública en estudio}

La universidad pública en estudio fue inaugurada en el año de 1965 y se encuentra ubicada en el sureste de México. Actualmente cuenta con nueve Facultades y una Escuela Superior donde se imparten 25 licenciaturas, ocho maestrías, seis especialidades médicas y un diplomado. Además, cuenta con seis centros de investigación, un instituto para conservar los recursos marino-costeros del país y un programa ambiental. Asimismo, esta universidad agrupa sus actividades educativas y de investigación en áreas afines que denomina Dependencias de Educación Superior (DES). En este sentido, la institución cuenta con cuatro DES: a) Ciencias Agropecuarias, b) Ciencias de la Salud, c) Ciencias Sociales y Humanidades, e d) Ingeniería y Ciencias. La DES de Ciencias Agropecuarias contempla la Escuela Superior de Ciencias Agropecuarias; la de Ciencias de la Salud agrupa a las Facultades de Enfermería, Medicina y Odontología; la de Ciencias Sociales y Humanidades considera las Facultades de Ciencias Sociales, Contaduría y Administración, Derecho y Humanidades; y la de Ingeniería y Ciencias 
integra las Facultades de Ingeniería y Ciencias Químico Biológicas. Cabe señalar que las DES integran, además de las Facultades señaladas, los diferentes centros de investigación, de acuerdo con sus áreas de estudio.

La capacidad emprendedora es una de las 15 competencias genéricas del perfil universitario para el nivel de licenciatura que esta universidad ha definido. Por tanto, la universidad cuenta con un Programa Institucional de Desarrollo de Emprendedores (PIDE), el cual tiene la finalidad de fortalecer la vinculación de toda la comunidad universitaria con los sectores productivos, de impulsar a los estudiantes a desarrollar sus competencias emprendedoras y de proporcionarles la orientación e información necesaria para el desarrollo de productos y servicios que deriven de empresas y así, contribuir al desarrollo económico del Estado.

\section{Investigaciones sobre competencias directivas}

Los estudios sobre la dirección de los centros educativos han sido amplios, de tal forma que pueden encontrarse investigaciones sobre problemas de las funciones directivas, el rol del director, la figura del equipo directivo, la distribución de las tareas, el liderazgo, el empowerment, las actitudes, la organización, el clima y la cultura, entre otros aspectos.

García, Poblete y Villa (2006) investigaron la evolución de la función directiva, encontrando cambios importantes en la formación tradicionalmente centrada en la figura del director y que, actualmente se realiza en torno al equipo directivo como órgano colegiado. Las últimas tendencias del estudio del liderazgo en los centros educativos, han pasado de centrarse en el puesto del director a un nuevo modelo llamado liderazgo distribuido, compartido o participativo.

García-Lombardía, Cardona y Chinchilla (2001) realizaron un estudio relativo a las competencias directivas más valoradas; los resultados indican que el aprendizaje, la toma de decisiones y la credibilidad son competencias muy valoradas para mejorar personalmente, y hacer frente a los cambios del siglo XXI.

Un estudio realizado por Maureira (2006) argumenta la relevancia que tiene el liderazgo como manifestación clave del rol de la dirección a través del tiempo y su relación con la eficacia escolar, así como las tendencias más recurrentes en la caracterización del liderazgo educativo. Además, señala que la dirección escolar constituye, cada vez más, un espacio clave para la mediación y dinamización de procesos docentes efectivos.

Bolívar (2010) encontró que el perfil del director de gestión se está ampliando a un liderazgo pedagógico. Un liderazgo para el aprendizaje, más allá de la dirección, se tiene que ampliar de modo distribuido, a través del liderazgo del profesorado y de las comunidades profesionales de aprendizaje.

Un trabajo realizado por Murillo (2006) indica que la investigación y la experiencia evidencian que el comportamiento y la actitud del individuo que asume las funciones de 
dirección en la escuela son factores fundamentales que determinan la existencia, la calidad y el éxito en los procesos de cambio en la escuela. Por lo tanto, el director debe ser una persona con las competencias necesarias para iniciar, impulsar, facilitar, gestionar y coordinar el proceso de cambio para la mejora de la calidad educativa.

\section{Metodología}

Este estudio es de tipo exploratorio y descriptivo, por lo que su diseño es no experimental (Hernández, Fernández y Baptista, 2010). Se utiliza como método un estudio de campo basado en cuatro casos seleccionados. Como se mencionó anteriormente, participan cuatro directores del área de ciencias sociales de una universidad pública ubicada en el sureste de México. Cada Facultad es considerada una unidad de análisis. Para identificar las competencias empleadas por dichos gerentes y la necesidad de adquirir otras con miras a mejorar la productividad escolar, se maneja la clasificación de competencias directivas o genéricas de Cardona y Chinchilla (1999) citadas en este trabajo.

Se contó con el apoyo de las cuatro Facultades de la universidad pública en estudio, así como también con la ayuda de cuatro estudiantes de licenciatura de dicha institución. Se llevaron a cabo entrevistas con cada uno de los directores de las Facultades y con el personal a su cargo. La recolección de datos incluyó observaciones de la jornada laboral durante una semana.

\section{Resultados}

A continuación, se presentan los resultados de cada unidad de análisis.

\section{Director 1.}

El director tiene 46 años de edad, es contador público y tiene una maestría en Alta Dirección. Cuenta con 24 años de experiencia profesional y seis años como director. Sus estudios están estrechamente relacionados con las actividades que realiza. Según los resultados de la entrevista, frecuénteme usa las competencias: visión de negocios, resolución de problemas y gestión de recursos. Señaló que, para tomar una decisión, primero obtiene información suficiente, analiza los factores internos y externos y elabora una síntesis de lo que puede ocurrir en múltiples dimensiones. Como universidad pública, cada año elabora la planeación de recursos financieros y, cada seis meses, la de recursos humanos. Señaló que debido a los múltiples usuarios se tienen contratiempos en la gestión escolary que debido a la diversidad de horarios no es posible tener una actividad de relaciones interpersonales todos los días, pero hay reuniones y juntas programadas. Mencionó que muy frecuentemente emplea la negociación mediante reuniones de profesores, logrando que los grupos importantes o claves se convenzan, participen y colaboren; añadió que es fácil porque los objetivos están formados en su conjunto.

Manifestó que la comunicación es frecuente debido a que los medios son diversos y no hay uno solo si se quiere dar a conocer algo. Sin embargo, comentó que es muy complicado saber si 
llegó la información a su destino, ya que no hay manera de asegurarse que la comunicación sea inmediata y tener evidencias para respaldar las opiniones. Asigna funciones a cada empleado y lleva un seguimiento del trabajo realizado para tomar medidas correctivas. Indicó que es muy difícil que la persona que tiene una necesidad vea la postura del otro; por ejemplo, un intendente que tiene la necesidad de salir solo ve su punto de vista como el correcto, pero no está pensando en la solución que se debe dar a la institución: ¿quién va a limpiar los pasillos?, ¿el baño?, ¿quién va a hacer ese trabajo?". Cada uno de sus colaboradores tiene la capacidad de operar y tomar decisiones hasta cierto nivel. Debido a que es una institución pública, cuando los subordinados requieren capacitación de sus funciones prefieren que la haga un tercero y no su jefe, por lo que el director solo intenta hacerlo cuando es necesario. En cuanto al trabajo en equipo, comentó que no se tiene un abanico de motivadores, no hay aumento de sueldo ni disminución de jornada laboral, pero sí se puede ofrecer buen trato, reconocimiento y horas continuas, entre otros.

Señaló ser proactivo, pues siempre hay que buscar una mejor manera de tener a su personal más contentos y ser más competitivos. Manifestó tener concentración y autocontrol, pero reconoció que le falta mayor disciplina. Comentó que frecuentemente utiliza la gestión personal, ya que le delegan ciertas responsabilidades y eso implica riesgos en la toma de decisiones; aunque aceptó que le falta un adecuado control del tiempo. Finalmente, señaló que se autoevalúa casi todos los días, ya que tiene muchas actividades y analiza si logró o no sus objetivos. Sin embargo, indicó que esta medición debería hacerla más estructurada.

\section{Colaboradores del Director 1.}

Los colaboradores perciben que el director frecuentemente emplea las competencias de visión de negocios, resolución de problemas, gestión de recursos y orientación al cliente. Sin embargo, le falta adquirir mayores competencias en red de relaciones efectivas y negociación. Los colaboradores señalaron que establece poca relación con personas que laboran en la Facultad y se le dificulta obtener la conformidad del personal. De acuerdo con la opinión de sus colaboradores, le falta mayor comunicación con los empleados y, aunque procura la buena organización y eficiencia del trabajo, le hace falta comprender a los demás. Muestra concentración e iniciativa en su trabajo. Además, tiene la habilidad para tomar las decisiones con el fin de alcanzar los resultados deseados. Sus colaboradores percibieron que no se autoevalúa lo suficiente para reconocer que puede mejorar y fortalecer el puesto que desempeña.

\section{Director 2.}

El director tiene 38 años de edad, es licenciado en economía con una maestría en economía y administración pública; cuenta con 13 años de experiencia profesional, de los cuales tres años se ha desempeñado como director académico. Señaló que solo algunas veces tiene visión de negocios, pues la institución no es una empresa. Frecuentemente resuelve problemas y trata de sacar buen provecho de los recursos que tiene asignados. Para responder a las necesidades del cliente se maneja un buzón de quejas y una encuesta de servicio, lo que le permite tomar 
medidas preventivas y correctivas. Se reúne, aunque no de manera frecuente, con su personal para tomar decisiones.

Logra el apoyo y la conformidad de su personal mediante escritos de recordatorio sobre atrasos en la entrega oportuna de documentos. Indicó que hace buen uso de la comunicación de manera frecuente; si existen problemas en los que tenga la razón, los enfrenta y aplica el coaching para que se den cuenta que está mal; además, muestra evidencias para respaldar sus puntos de vista y busca la mejor opción para salir adelante. Manifestó que asigna funciones a su personal mediante reuniones, donde se dice que se va a realizar y a través de oficios o escrito les da seguimiento. En cuanto a la empatía, señaló que no hay tiempo de enterarse de los sentimientos de los demás, pues la única forma de saberlo es platicando y no tiene mucho tiempo para hacerlo. Delega frecuentemente tareas a sus colaboradores inmediatos y ellos se coordinan con su personal subordinado para realizar el trabajo; no obstante, para él los responsables siguen siendo sus colaboradores inmediatos. Apoya a sus colaboradores cuando se atrasan en las encomiendas, tienen que mejorar una parte más que la otra, o bien, cuando se valora más lo prioritario sobre lo emergente. Comentó que promueve un clima de colaboración y motiva a su equipo de trabajo a través de reuniones, ya que no depende todo de él.

Indicó que usa la proactividad, pues ha celebrado convenios con otras universidades que le han permitido que seis maestros obtengan becas para estudios de diplomado. Comentó que ha aprendido a controlarse con el tiempo y procura tener disciplina en los horarios y control emocional, ya que pueden generar un desempeño bueno o malo. Practica deportes de gimnasia y camina para poder liberar la presión que tiene. No se autoevalúa, pero indicó que los califican y eso le ayuda a darse cuenta qué parte está débil para tener cambios en la eficiencia y tomar decisiones, aceptando sus errores con humildad.

\section{Colaboradores del Director 2.}

Los colaboradores perciben que el director frecuentemente emplea las competencias resolución de problemas y gestión de recursos, pero le falta mayor visión de negocio, responder en tiempo eficaz a las necesidades de los clientes, incrementar su red de relaciones efectivas y más capacidad de negociación. Los colaboradores manifiestan que el director mantiene buena comunicación, tiene en cuenta a los demás, ayuda a sus colaboradores y promueve un clima de colaboración, pero le falta mayor organización y delegación. Tiene iniciativa y es disciplinada, empero le falta mayor gestión personal y autoevaluarse para lograr cambios que fortalezcan su gestión personal.

\section{Director 3.}

El director tiene 43 años de edad, es licenciado en derecho y tiene estudios de maestría y doctorado, cuenta con 19 años de experiencia profesional, de los cuales seis años se ha desempeñado como director académico. Especificó que tiene visión de negocio, capacidad para reconocer problemas complejos, hacer buen uso de los recursos asignados y capacidad 
de negociación porque su experiencia le proporciona la fortaleza para ser competitivo en su trabajo y en razón de la economía, logra alcanzar en un menor tiempo los objetivos trazados. Reconoció que le falta mayor orientación al cliente e incrementar su red de relaciones efectivas para aumentar sus oportunidades de desarrollo.

Mencionó que tiene capacidad de comunicación, encarga funciones específicas a determinadas personas, dando como resultado una adecuada atención a las necesidades requeridas. Logra la realización profesional del personal a su cargo mediante la capacitación, la libertad que otorga en el desempeño del trabajo y en la toma de decisiones; con esto logra fortalecer el trabajo en equipo. Por otra parte, comentó que le falta usar mayor empatía para comprender mejor a los demás.

Aclaró que es proactivo, pues el liderazgo que proyecta al interior de su personal propicia la creatividad e iniciativa de su personal. Comentó que, en la medida de lo posible, procura tener control ante los retos o riesgos que pretenden desestabilizar el buen logro de los objetivos trazados y que, frecuentemente, se autoevalúa de manera honesta reconociendo sus propias fortalezas y debilidades, en aras de alcanzar sus metas y objetivos.

\section{Colaboradores del Director 3.}

Los colaboradores indicaron que el director frecuentemente emplea las competencias visión de negocios, resolución de problemas, gestión de recursos, orientación al cliente y negociación, pero le falta ampliar su red de relaciones efectivas. Aunque tiene una adecuada organización, empatía, delegación y coaching, le falta mayor comunicación y trabajo en equipo. Muestra iniciativa, tiene control de la situación, pero le falta autoevaluarse.

\section{Director 4.}

El director tiene 50 años de edad, cuenta con una licenciatura en psicología y tiene un posgrado con nivel de maestría en ciencias de la educación, cuenta con 16 años de experiencia profesional en el campo de la educación. Indicó que tiene buena visión de negocios, pues ha sabido gestionar los recursos de manera adecuada y ampliarlos a un largo plazo, para alcanzar objetivos programados a futuro, aun cuando no esté presente. Mencionó que durante su gestión se han presentado muchas discrepancias entre alumnos, maestros y personal administrativo, teniendo que elegir el mejor juicio profesional para abordar la problemática desde un punto de vista objetivo y darle solución al problema de manera imparcial, aplicando las reglas cuando se requieren. Comentó que, gracias a su gestión, hasta ahora no ha existido problema alguno que se salga de control, pues los alumnos y el personal se acercan para expresar sus inconformidades y toma cartas en el asunto para que este malestar no pase a mayores. Cuando detecta una oportunidad que sea benéfica para la Facultad, se acerca a ella y no la deja pasar, pues considera que contribuye al crecimiento de la institución. Considera que a veces se presentan situaciones en las que se deben tomar decisiones asertivas para la salud institucional, por lo que hay que saber persuadir y convencer a las personas sobre las decisiones tomadas por 
la directiva. Asimismo, destacó que, algunas veces, por cuestiones externas y en cumplimiento de sus obligaciones para su campo de trabajo, le es difícil cumplir del todo las necesidades de los alumnos y la plantilla docente.

En relación con la comunicación, considera que se cumple de buena manera, pues nunca se ha suscitado incertidumbre, error o falla en la elaboración de las tareas por falta de comunicación. Comentó que da seguimiento constante a las tareas que le encomienda a su personal, pues piensa que si quiere que las cosas salgan como se establecen es necesario estar pendiente de ellas en todo momento, para evitar que se desvíen de los objetivos con los que se trabaja y vigilar su cumplimiento. Expresó que el director no debe ser autocrático, es decir, no debe abusar de su posición en la estructura jerárquica de la institución, pues esto genera descontentos entre el personal; debe tener en cuenta las ideas de la gente y respetarlas, ganarse a las personas generando un buen ambiente organizacional. Mencionó que, a veces, por el exceso de actividades a realizar, es necesario delegar funciones, ya que esto involucra confiar en las capacidades de las personas encaminadas al logro de objetivos, así como confiar que las decisiones que tomen serán las más acertadas. Señaló que le hace falta ayudar a sus colaboradores a mejorar y desarrollar sus habilidades, pero hace todo lo posible por lograr este rubro. Además, le falta motivar, de manera más efectiva, a su personal.

Manifestó que siempre muestra iniciativa, creatividad y autonomía personal e implementa la disciplina como parte de su concentración y autocontrol con el personal. Dijo que es necesario hacer una introspectiva para consigo misma, pues esto le permite autoevaluarse de qué tan bien se está desenvolviendo en el campo, esto con el fin de que fortalezca su gestión directiva; también indicó que este tipo de entrevistas le permiten mejorar su gestión.

\section{Colaboradores del Director 4.}

Los colaboradores comentaron que el director frecuentemente utiliza las competencias de visión de negocios, resolución de problemas, gestión de recursos, red de relaciones efectivas y negociación, pero le falta mayor orientación al cliente. Tiene buena organización, delegación y promueve el trabajo en equipo; le falta mayor comunicación, empatía y ayudar más a sus colaboradores a mejorary a desarrollar sus habilidades y capacidades. Aunque tiene autocontrol y gestión personal, le falta mayor iniciativa y autoevaluación.

\section{Discusión}

Todos los participantes coinciden en que los directores usan la resolución de problemas y la gestión de recursos. Asimismo, de acuerdo con la percepción de los subordinados, todos los directores requieren autoevaluarse para fortalecer su gestión directiva y por tanto, sus competencias de desarrollo personal.También, resultó que solamente el director 2 mantiene una adecuada comunicación, y el director 4 desempeña una apropiada red de relaciones efectivas, por lo que es necesario que los demás mejoren estas competencias. Mientras que los directores 
señalan que manejan un mayor número de competencias, los subordinados no lo perciben así, ya que indican que les falta adquirir varias de las competencias señaladas por los directores como utilizables, tales como orientación al cliente, red de relaciones efectivas, negociación, comunicación, empatía, trabajo en equipo y desarrollo personal. De manera específica, las competencias estratégicas usadas y las que necesitan desarrollarse pueden apreciarse en las tablas 1 y 2; las competencias intratégicas, en las tablas 3 y 4 ; y las competencias de eficacia personal, en las tablas 5 y 6.

\section{Tabla 1}

Competencias estratégicas utilizadas y por adquirir de los directores académicos

\begin{tabular}{|c|c|c|c|c|c|c|}
\hline \multirow[t]{2}{*}{ Competencia } & \multirow{2}{*}{$\begin{array}{c}\text { Director 1/ } \\
\text { Subordinados }\end{array}$} & \multirow{2}{*}{$\begin{array}{c}\text { Director 2/ } \\
\text { Subordinados }\end{array}$} & \multirow{2}{*}{$\begin{array}{c}\text { Director 3/ } \\
\text { Subordinados }\end{array}$} & \multirow{2}{*}{$\begin{array}{c}\text { Director 4/ } \\
\text { Subordinados }\end{array}$} & \multicolumn{2}{|c|}{ Total \% } \\
\hline & & & & & $\begin{array}{l}\mathrm{D} / \mathrm{S} \\
\text { Utiliza }\end{array}$ & $\begin{array}{c}\mathrm{D} / \mathrm{S} \\
\text { Adquirir }\end{array}$ \\
\hline $\begin{array}{l}\text { Visión de nego- } \\
\text { cio }\end{array}$ & $\mathrm{U} / \mathrm{U}$ & $\mathrm{A} / \mathrm{A}$ & $\mathrm{U} / \mathrm{U}$ & $\mathrm{U} / \mathrm{U}$ & $75 / 75$ & $25 / 25$ \\
\hline $\begin{array}{l}\text { Resolución de } \\
\text { problemas }\end{array}$ & $\mathrm{U} / \mathrm{U}$ & $\mathrm{U} / \mathrm{U}$ & $\mathrm{U} / \mathrm{U}$ & $\mathrm{U} / \mathrm{U}$ & $100 / 100$ & \\
\hline $\begin{array}{l}\text { Gestión de } \\
\text { recursos }\end{array}$ & $\mathrm{U} / \mathrm{U}$ & $\mathrm{U} / \mathrm{U}$ & $\mathrm{U} / \mathrm{U}$ & $\mathrm{U} / \mathrm{U}$ & $100 / 100$ & \\
\hline $\begin{array}{l}\text { Orientación al } \\
\text { cliente }\end{array}$ & $\mathrm{U} / \mathrm{U}$ & $\mathrm{U} / \mathrm{A}$ & $A / U$ & $\mathrm{~A} / \mathrm{A}$ & $50 / 50$ & $50 / 50$ \\
\hline $\begin{array}{l}\text { Red de relacio- } \\
\text { nes efectivas }\end{array}$ & $U / A$ & $U / A$ & $A / A$ & $\mathrm{U} / \mathrm{U}$ & $75 / 25$ & $25 / 75$ \\
\hline Negociación & $\mathrm{U} / \mathrm{A}$ & $U / A$ & $\mathrm{U} / \mathrm{U}$ & $\mathrm{U} / \mathrm{U}$ & $100 / 50$ & $0 / 50$ \\
\hline
\end{tabular}

Nota: Elaborada a partir de datos recolectados por los investigadores. Simbología: U: utiliza, A: falta adquirir.

En las tablas 1 y 2 se advierte que, tanto los directores como sus subordinados, coinciden en que usan la resolución de problemas y la gestión de recursos (100\%), pero que les falta adquirir mayores competencias en visión de negocios (25\%) y orientación al cliente (50\%). Aunque los directores dicen que emplean la red de relaciones efectivas (75\%) y la negociación (100\%), los subordinados perciben que les falta adquirir estas competencias ( $75 \%$ y $50 \%$, respectivamente). 
Rev. Gestión de la Educación, Vol. 6, N², [1-18], ISSN: 2215-2288, julio-diciembre, 2016

DOI: http://dx.doi.org/10.15517/rge.v1i2.25479

URL: http://revistas.ucr.ac.cr/index.php/gestedu

\section{Tabla 2}

Categorización de competencias estratégicas de los directores académicos percibidas por sus subordinados

\begin{tabular}{|c|c|c|c|c|c|}
\hline \multirow{2}{*}{$\begin{array}{l}\text { Competencia } \\
\text { Categoría }\end{array}$} & \multirow[t]{2}{*}{ Unidades } & \multicolumn{2}{|c|}{ Director } & \multicolumn{2}{|c|}{ Total \% } \\
\hline & & Utiliza & Adquirir & Utiliza & Adquirir \\
\hline \multirow[t]{3}{*}{$\begin{array}{l}\text { Visión de } \\
\text { negocio }\end{array}$} & $\begin{array}{l}\text { "Identifica las amenazas y hace buen uso de las } \\
\text { oportunidades que se le presentan". }\end{array}$ & 1,3 & & 75 & \\
\hline & $\begin{array}{l}\text { "Tiene conocimientos y aplica la planeación } \\
\text { estratégica" }\end{array}$ & 4 & & & \\
\hline & "Le falta ver más allá de lo que otros ven" & & 2 & & 25 \\
\hline \multirow[t]{2}{*}{$\begin{array}{l}\text { Resolución de } \\
\text { problemas }\end{array}$} & $\begin{array}{l}\text { "Analiza los pros y los contras ante una situación } \\
\text { dada" }\end{array}$ & 1,3 & & 100 & \\
\hline & $\begin{array}{l}\text { "Ha logrado resolver problemas sencillos y } \\
\text { complejos" }\end{array}$ & 2,4 & & & \\
\hline \multirow[t]{2}{*}{$\begin{array}{l}\text { Gestión de } \\
\text { recursos }\end{array}$} & $\begin{array}{l}\text { "Supervisa la asignación racional de los recursos } \\
\text { para la adecuada operatividad" }\end{array}$ & 1,3 & & 100 & \\
\hline & $\begin{array}{l}\text { "Procura proveer los recursos para el cumplimien- } \\
\text { to de las tareas" }\end{array}$ & 2,4 & & & \\
\hline \multirow{2}{*}{$\begin{array}{l}\text { Orientación al } \\
\text { cliente }\end{array}$} & "Muestra cortesía en la atención a los estudiantes" & 1,3 & & 50 & \\
\hline & $\begin{array}{l}\text { "Requiere estar más pendiente de las exigencias } \\
\text { de las personas" }\end{array}$ & & 2,4 & & 50 \\
\hline \multirow[t]{2}{*}{$\begin{array}{l}\text { Red de relacio- } \\
\text { nes efectivas }\end{array}$} & $\begin{array}{l}\text { "Establece un trato asertivo con todas las perso- } \\
\text { nas" }\end{array}$ & 4 & & 25 & \\
\hline & $\begin{array}{l}\text { "Se relaciona muy poco con los profesores y el } \\
\text { personal" }\end{array}$ & & $1,2,3$ & & 75 \\
\hline \multirow[t]{2}{*}{ Negociación } & $\begin{array}{l}\text { "Siempre busca la forma de convencer a sus } \\
\text { colaboradores" }\end{array}$ & 3.4 & & 50 & \\
\hline & $\begin{array}{l}\text { "En ocasiones la diversidad de opiniones le difi- } \\
\text { culta llegar a acuerdos y conseguir apoyo" }\end{array}$ & & 1.2 & & 50 \\
\hline
\end{tabular}

Nota: Elaborada con datos recolectados por los investigadores, basados en Hernández, Fernández y Baptista (2010). 
Tabla 3

\section{Competencias intratégicas utilizadas y por adquirir de los directores académicos}

\begin{tabular}{|c|c|c|c|c|c|c|}
\hline \multirow[t]{2}{*}{ Competencia } & \multirow{2}{*}{$\begin{array}{c}\text { Director 1/ } \\
\text { Subordinados }\end{array}$} & \multirow{2}{*}{$\begin{array}{r}\text { Director 2/ } \\
\text { Subordinados }\end{array}$} & \multirow{2}{*}{$\begin{array}{c}\text { Director 3/ } \\
\text { Subordinados }\end{array}$} & \multirow{2}{*}{$\begin{array}{c}\text { Director 4/ } \\
\text { Subordinados }\end{array}$} & \multicolumn{2}{|c|}{ Total \% } \\
\hline & & & & & $\begin{array}{c}\text { D/S } \\
\text { Utiliza }\end{array}$ & $\begin{array}{c}\mathrm{D} / \mathrm{S} \\
\text { Adquirir }\end{array}$ \\
\hline Comunicación & U/A & $\mathrm{U} / \mathrm{U}$ & $\mathrm{U} / \mathrm{A}$ & U/A & $100 / 25$ & $0 / 75$ \\
\hline Organización & $\mathrm{U} / \mathrm{U}$ & $\mathrm{U} / \mathrm{A}$ & $\mathrm{U} / \mathrm{U}$ & $\mathrm{U} / \mathrm{U}$ & $100 / 75$ & $0 / 25$ \\
\hline Empatía & $\mathrm{U} / \mathrm{A}$ & $A / U$ & $\mathrm{~A} / \mathrm{U}$ & $\mathrm{U} / \mathrm{A}$ & $50 / 50$ & $50 / 50$ \\
\hline Delegación & $\mathrm{U} / \mathrm{U}$ & $\mathrm{U} / \mathrm{A}$ & $\mathrm{U} / \mathrm{U}$ & $\mathrm{U} / \mathrm{U}$ & $100 / 75$ & $0 / 25$ \\
\hline Coaching & $\mathrm{U} / \mathrm{U}$ & $\mathrm{U} / \mathrm{U}$ & $\mathrm{U} / \mathrm{U}$ & $A / A$ & $75 / 75$ & $25 / 25$ \\
\hline $\begin{array}{l}\text { Trabajo en } \\
\text { equipo }\end{array}$ & $\mathrm{U} / \mathrm{A}$ & $\mathrm{U} / \mathrm{U}$ & $\mathrm{U} / \mathrm{A}$ & $\mathrm{A} / \mathrm{U}$ & $75 / 50$ & $25 / 50$ \\
\hline
\end{tabular}

Nota: Elaborada a partir de datos recolectados por los investigadores. Simbología: U: utiliza, A: falta adquirir.

\section{Tabla 4}

Categorización de competencias intratégicas de los directores académicos percibidas por sus subordinados

\begin{tabular}{|c|c|c|c|c|c|}
\hline \multirow{2}{*}{$\begin{array}{l}\text { Competencia } \\
\text { Categoría }\end{array}$} & \multirow[t]{2}{*}{ Unidades } & \multicolumn{2}{|c|}{ Director } & \multicolumn{2}{|c|}{ Total \% } \\
\hline & & Utiliza & Adquirir & Utiliza & Adquirir \\
\hline \multirow[t]{2}{*}{ Comunicación } & "Lo que dice lo respalda y lo comprueba" & 2 & & 25 & \\
\hline & "Falta mejorar los canales de comunicación" & & $1,3,4$ & & 75 \\
\hline \multirow[t]{2}{*}{ Organización } & "Sabe ubicar a las personas de acuerdo a su perfil" & $1,3,4$ & & 75 & \\
\hline & "Las tareas no siempre se realizan como debiera" & & 2 & & 25 \\
\hline \multirow[t]{2}{*}{ Empatía } & $\begin{array}{l}\text { "Frecuentemente platica con el personal lo que le ha per- } \\
\text { mitido entenderlos y respetarlos" }\end{array}$ & 2,3 & & 50 & \\
\hline & "Le hace falta comprender a los demás" & & 1,4 & & 50 \\
\hline \multirow[t]{2}{*}{ Delegación } & $\begin{array}{l}\text { "No deja que el personal tome decisiones y toda la respon- } \\
\text { sabilidad recae en él" }\end{array}$ & $1,3,4$ & & 75 & \\
\hline & $\begin{array}{l}\text { "Le falta delegar mayor autoridad a su personal para la reso- } \\
\text { lución de problemas" }\end{array}$ & & 2 & & 25 \\
\hline \multirow[t]{2}{*}{ Coaching } & $\begin{array}{l}\text { "Identifica las habilidades de cada empleado y busca su } \\
\text { mejora" }\end{array}$ & $1,2,3$ & & 75 & \\
\hline & $\begin{array}{l}\text { "Necesita lograr que todos los empleados estén dispuestos a } \\
\text { cooperar" }\end{array}$ & & 4 & & 25 \\
\hline Competencia & Unidades & Director & & & al \% \\
\hline Categoría & & Utiliza & Adquirir & Utiliza & Adquirir \\
\hline \multirow[t]{2}{*}{ Trabajo en equipo } & $\begin{array}{l}\text { "Cuando un empleado está ocupado en alguna actividad, } \\
\text { otro lo apoya sacando adelante el trabajo" }\end{array}$ & 2,4 & & 50 & \\
\hline & $\begin{array}{l}\text { "Falta apoyo mutuo y confianza para una mejor integración } \\
\text { de equipo" }\end{array}$ & & 1,3 & & 50 \\
\hline
\end{tabular}

Nota: Elaborada con datos recolectados por los investigadores, basados en Hernández, Fernández y Baptista (2010). 
En las tablas 3 y 4 se observa que, tanto los directores como sus subordinados, coinciden en que les falta adquirir la competencia de empatía (50\%). Mientras que los directores señalan que manejan las competencias de comunicación, organización y delegación (100\%), los subordinados perciben que les falta adquirirlas ( $75 \%, 25 \%$ y $25 \%$, respectivamente). Asimismo, los directores indican que usan el coaching y el trabajo en equipo (75\%), pero sus subordinados manifiestan que requieren adquirir estas competencias ( $25 \%$ y $50 \%$, respectivamente).

\section{Tabla 5}

\section{Competencias de eficacia personal, utilizadas y por adquirir de los directores académicos}

\begin{tabular}{lcccccc}
\hline Competencia & $\begin{array}{c}\text { Director 1/ } \\
\text { Subordinados }\end{array}$ & $\begin{array}{c}\text { Director 2/ } \\
\text { Subordinados }\end{array}$ & $\begin{array}{c}\text { Director 3/ } \\
\text { Subordinados }\end{array}$ & $\begin{array}{c}\text { Director 4/ } \\
\text { Subordinados }\end{array}$ & & \multicolumn{2}{c}{$\begin{array}{c}\text { Total } \% \\
\text { Utiliza }\end{array}$} & $\begin{array}{c}\text { D/S } \\
\text { Adquirir }\end{array}$ \\
\hline Proactividad & $\mathrm{U} / \mathrm{U}$ & $\mathrm{U} / \mathrm{U}$ & $\mathrm{U} / \mathrm{U}$ & $\mathrm{U} / \mathrm{A}$ & $100 / 75$ & $0 / 25$ \\
Autogobierno & $\mathrm{U} / \mathrm{U}$ & $\mathrm{U} / \mathrm{U}$ & $\mathrm{A} / \mathrm{U}$ & $\mathrm{U} / \mathrm{U}$ & $75 / 100$ & $25 / 0$ \\
Gestión personal & $\mathrm{U} / \mathrm{U}$ & $\mathrm{U} / \mathrm{A}$ & $\mathrm{U} / \mathrm{U}$ & $\mathrm{U} / \mathrm{U}$ & $100 / 75$ & $0 / 25$ \\
$\begin{array}{l}\text { Desarrollo per- } \\
\text { sonal }\end{array}$ & $\mathrm{U} / \mathrm{A}$ & $\mathrm{U} / \mathrm{A}$ & $\mathrm{U} / \mathrm{A}$ & $\mathrm{A} / \mathrm{A}$ & $75 / 0$ & $25 / 100$ \\
\hline
\end{tabular}

Nota: Elaborada a partir de datos recolectados por los investigadores. Simbología: U: utiliza, A: falta adquirir

Tabla 6

Categorización de competencias de eficacia personal de los directores académicos percibidas por sus subordinados

\begin{tabular}{|c|c|c|c|c|c|}
\hline \multirow{2}{*}{$\begin{array}{l}\text { Competencia } \\
\text { Categoría }\end{array}$} & \multirow[t]{2}{*}{ Unidades } & \multicolumn{2}{|c|}{ Director } & \multicolumn{2}{|c|}{ Total \% } \\
\hline & & Utiliza & Adquirir & Utiliza & Adquirir \\
\hline \multirow[t]{4}{*}{ Proactividad } & "Crea proyectos de atracción al cliente con buenos resultados" & 1 & & 75 & \\
\hline & "La cuestión es renovarse o morir" & 2 & & & \\
\hline & personal" & 3 & & & \\
\hline & $\begin{array}{l}\text { "Le falta más empuje y vivacidad para solucionar algunos } \\
\text { problemas" }\end{array}$ & & 4 & & 25 \\
\hline Autogobierno & $\begin{array}{l}\text { "Muestra disciplina y enfoca los problemas sin perder de vista la } \\
\text { normatividad y la reglamentación" }\end{array}$ & $1,2,3,4$ & & 100 & \\
\hline Competencia & \multirow[t]{2}{*}{ Unidades } & \multicolumn{2}{|c|}{ Director } & \multicolumn{2}{|c|}{ Total \% } \\
\hline Categoría & & Utiliza & Adquirir & Utiliza & Adquirir \\
\hline \multirow[t]{2}{*}{ Gestión personal } & $\begin{array}{l}\text { "Es muy cumplido y puntual con los tiempos establecidos, no } \\
\text { deja para lo último los pendientes" }\end{array}$ & $1,3,4$ & & 75 & \\
\hline & $\begin{array}{l}\text { "En ocasiones ciertos trabajos no se entregan en el tiempo } \\
\text { establecido y se solicita prorroga" }\end{array}$ & & 2 & & 25 \\
\hline \multirow{2}{*}{$\begin{array}{l}\text { Desarrollo per- } \\
\text { sonal }\end{array}$} & "Siempre está dispuesto a mejorar" & 3 & & 25 & \\
\hline & "No se nota un cambio de actitud, su comportamiento es el mismo" & & $1,2,4$ & & 75 \\
\hline
\end{tabular}

Nota: Elaborada con datos recolectados por los investigadores, basados en Hernández, Fernández y Baptista (2010). 
En las tablas 5 y 6 se detalla que, mientras los directores refieren que utilizan las competencias de proactividad y gestión personal (100\%), sus subordinados mencionan que les falta adquirir esas competencias (25\%). Asimismo, los directores indicaron que usan las competencias de autogobierno y desarrollo personal (75\%), pero que les falta adquirirlas un poco más (25\%); mientras que sus subordinados perciben que cuentan con las competencias de autogobierno (100\%) y les falta adquirir las de desarrollo personal (100\%).

\section{Conclusiones}

Existen diferencias entre conocimientos y competencias, pues aunque los directores tienen la preparación que les permite estudiar y comprender aspectos relacionados con la educación superior, se les dificulta al momento de aplicarlos. Esto es consistente con McClelland (1975), citado por Gross (2008), quien señala que desempeñar bien el trabajo depende de las características propias de la persona, es decir, más de sus competencias que de sus conocimientos.

Los directores manejan las competencias estratégicas de visión de negocios, resolución de problemas y gestión de recursos, pues tienen definida su visión, analizan sus problemas de manera sistemática y usan los recursos de forma racional. Sin embargo, las competencias orientación al cliente, red de relaciones efectivas y negociación demostraron ser más complejas en función de su desempeño efectivo. En cuanto a las competencias intratégicas, la complejidad en su desempeño la manifestaron, en mayor grado, la comunicación, la empatía y el trabajo en equipo y, en menor grado, la organización, la delegación y el coaching. En relación con las competencias de eficacia personal, los cuatro directores presentaron dificultades para utilizar las competencias de desarrollo personal, y en menor grado, las competencias de proactividad, autogobierno y gestión personal. Por tanto, es necesario que los directores se autoevalúen para lograr mejoras en su comportamiento y en su gestión directiva.

Los directores usan más las competencias requeridas para el logro de resultados y la unidad interna de la institución, y usan menos las que se refieren a la relación eficaz de la persona consigo misma y con el entorno. En términos generales, los directores requieren adquirir y desarrollar competencias en las tres categorías, principalmente en las áreas del comportamiento humano, lo que es congruente con Chávez (2013), quien indica que es importante que el directivo tome la iniciativa para desarrollar y perfeccionar habilidades con las que pueda mejorar su nivel competitivo.

En cuanto a las áreas del comportamiento humano, los directores señalaron que no tienen tiempo para establecer relaciones interpersonales o enterarse de los sentimientos de los demás. En este sentido, los directivos deben desarrollar y mantener relaciones humanas efectivas, tanto con el personal de su Facultad como con los alumnos y las demás personas clave del entorno en el que se desenvuelven, de tal manera que les permita alcanzar los objetivos establecidos, de manera satisfactoria. Esto es consistente con Murillo (2006), quien resaltando la importancia 
que tienen las personas sobre los papeles y las tareas, manifiesta que un cambio positivo solo es posible mediante el buen humor y las buenas relaciones humanas, pues de esta forma se logra mayor implicación, compromiso y trabajo en equipo.

Se recomienda el desarrollo de estudios relacionados con el tema, que muestren aspectos cuantitativos. Asimismo, se propone diseñar una intervención en otras instituciones de educación superior y elaborar estudios comparativos. También, podría investigarse sobre las competencias y su influencia en los estilos de dirección, el ambiente y la cultura organizacional, entre otros aspectos, en beneficio de la mejora de las competencias directivas escolares y de la comunidad educativa.

\section{Referencias}

Bolívar, A. (2010). El liderazgo educativo y su papel en la mejora: una revisión actual de sus posibilidades y limitaciones. Psicoperspectivas. Individuo y Sociedad, 9(2), 9-33. Recuperado de: http://www.redalyc.org/pdf/1710/171015625002.pdf

Cardona, P. y Chinchilla, M. N. (1999). Evaluación y desarrollo de las competencias directivas. Harvard Deusto Business Review, 89, 10-27. Recuperado de: https://dialnet.unirioja.es/ servlet/articulo?codigo $=63273$

Chávez, N. (2013). Una aproximación a las competencias gerenciales elementales. Gerencia y Negocios en Hispanoamérica, De gerencia.com. Recuperado de: http://www.degerencia. com/articulo/una-aproximacion-a-las-competencias-gerenciales-elementales

Elizondo, L. (2011). Competencias que debe tener un director académico universitario para la educación superior basada en competencias. Revista de Investigación Educativa, 29(1), 205-218. Recuperado de: http://revistas.um.es/rie/article/view/110791/127042

García-Lombardía, P., Cardona, P. y Chinchilla, M. (2001). Las competencias directivas más valoradas. IESE Business School, Occasional Paper, 1(4), 1-24. Recuperado de: http://www. iese.edu/research/pdfs/op-01-04.pdf

García, A., Poblete, M. y Villa, A. (2006). La función directiva: un problema sin resolver. Tres décadas de formación, investigación y acción. Revista de Educación, 8, 13-34. Recuperado de: http:// rabida.uhu.es/dspace/bitstream/handle/10272/2011/b15167227.pdf?sequence=1

Gross, M. (2008). Nuestro déficit de competencias directivas (corregido). Pensamiento imaginactivo. Recuperado de: http://manuelgross.bligoo.com/content/view/161409/ Nuestro-deficit-de-Competencias-Directivas-corregido.htm

Hernández, R., Fernández, C. y Baptista, P. (2010). Metodología de la investigación. México: Mc Graw Hill. 
Maureira, O. (2006). Dirección y eficacia escolar, una relación fundamental. Revista lberoamericana sobre Calidad, Eficacia y Cambio en Educación, 4(4e), 1-10. Recuperado de: http://www. redalyc.org/pdf/551/55140402.pdf

Murillo, F. J. (2006). Una dirección escolar para el cambio: del liderazgo transformacional al liderazgo distribuido. Revista lberoamericana sobre Calidad, Eficacia y Cambio en Educación, 4 (4e), 11-24. Recuperado de: http://www.rinace.net/arts/vol4num4e/art2.pdf

Poder Ejecutivo Federal. (1996). Programa de desarrollo educativo 1995-2000. México: Secretaría de Educación Pública.

Robbins, S. y Judge, T. (2013). Comportamiento organizacional. México: Pearson-Prentice Hall.

Sammons, P., Hillman, J. y Mortimer, P. (1995). Key characteristics of effective school: A review of school effectiveness research. London: OFSED and Institute of Education.

Sandoval, J. y Unda, S. (2004). Trabajo precario y salud en los docentes. Gaceta Académica del Sindicato Independiente de Trabajadores de la Universidad Autónoma Metropolitana. México: SITUAM.

Spencer, L. M. \& Spencer, S. M. (1993). Competence at work. Models for superior performance. New York: JohnWiley \& Sons.

Uribe, M. (2007). Liderazgo y competencias directivas para la eficacia escolar: experiencia del modelo de gestión escolar de Fundación Chile. Revista Iberoamericana sobre Calidad, Eficacia y Cambio en Educación, 5(5e), 149-156. Recuperado de: http://www.redalyc.org/ articulo.oa?id=55121025022

Villa, A. (2006). Elementos significativos de la loce con relación a las competencias directivas. Recuperado de: www.eyg.es/viicongreso 